\section{Humman Heredity}

\begin{tabular}{|c|c|}
\hline Amos, C.I. 1, 107, 139 & $\begin{array}{l}\text { Dietter, J. } 146 \text { (A) } \\
\text { Do, K.-A. } 146 \text { (A) }\end{array}$ \\
\hline Basu, S. 119 & \\
\hline Baur, M.P. 1 & Fürst, R. 41 \\
\hline Becker, T. 2 & \\
\hline Briollais, L. 94 & Goldgar, D. 48 \\
\hline Bull, S.B. 94 & Gorlova, O.Y. 139 \\
\hline Cannings, C. 126 & Hebebrand, J. 56 \\
\hline Chapman, J.M. 18 & Henschke, C. 139 \\
\hline Chaudru, V. 147 (A) & \\
\hline Chen, J. 107 & Kimmel, M. 139 \\
\hline Clayton, D.G. 18 & Knapp, M. 2 \\
\hline Conti, D.V. 32,83 & König, I.R. 63 \\
\hline Cooper, J.D. 18 & \\
\hline Cortessis, V. 83 & Lange, C. 10 \\
\hline & Lei, L. 139 \\
\hline Demenais, F. 147 (A) & Logan, A.G. 94 \\
\hline DeMeo, D. 10 & Lyon, H. 10 \\
\hline Dempfle, A. 56 & \\
\hline
\end{tabular}

$(\mathrm{A})=$ Abstract

\author{
Marjoram, P. 32 \\ Mirea, L. 94 \\ Molitor, J. 32, 83 \\ Moore, J.H. 73 \\ Mueller, P. 146 (A) \\ Müller, H.-H. 56
}

Pritchard, J. 149 (A)

Raby, B. 10

Rohde, K. 41

Rosenberg-Bourgin, M. 147 (A)

Schäfer, H. 1, 56

Scherag, A. 56

Shete, S. 107

Silverman, E.K. 10

Spitz, M. 139

Stram, D. 48

Stram, D.O. 32

Strauch, K. 146 (A)
Tang, F. 146 (A)

Thomas, D.C. 32,83

Thompson, D. 48

Thompson, E. 119

Todd, J.A. 18

Warwick Daw, E. 147 (A)

Wei, Q. 139

Weiss, S.T. 10

Witte, J.S. 48

Wu, X. 139

Yu, R.K. 107

Zhao, H. 131

Ziegler, A. 63

Zöllner, S. 149 (A)

Zou, G. 131

Subject Index Vol. 56, No. 1-3, 2003

Adaptive designs 56

Association 48

- analysis 2

- studies 18

- $\quad$-, family based 56

Bayesian methods 32

- model averaging 83

Biological systems moduling 73

Candidate gene associations 32

Case-control studies 32, 63

Case-parent trios 56

Conditional rejection probability 56

Design modifications 56

DNA repair capacity 139

Family-based association tests 10

Family data 131

Gene identification 107

Gene-environment interaction(s) 83, 94

Gene-gene interaction(s) 73, 83, 94
Genetic association 63

- heterogeneity 94

- susceptibility 139

Genome 126

Genotyping errors 131

Haplotype(s) 2, 32, 48

- estimation 41

- frequency 131

Identity by descent 119,126

Inheritance vector 119

Likelihood ratio test 94

Linkage analysis 94

- disequilibrium 18

- - mapping 32

Locus heterogeneity 94

Lung cancer 139

Markov chain(s) 107, 126

- - Monte Carlo Technique 41, 119

Mass screening, lung cancer 139

Metabolic pathways 83
Multifactor dimensionality reduction 73

Multi-locus SNP haplotypes 41

- TDT 18

Multiple comparisons 83

- imputation 119

Mutagen sensitivity 139

Parameter constraints 94

Pharmacokinetic models 83

Population data 131

Quantitative traits 10

Sample size reassessment 56

Sequential study design 63

Single nucleotide polymorphisms 32,48 , 107

- $\quad$ - - , haplotype tag 18

Smoking history, lung cancer 139

Spatial statistics 32

Testing strategies 10

Two-sample t test 94

\title{
KARGER
}

(C) 2003 S. Karger AG, Basel

Fax +4161306 1234

E-Mail karger@karger.ch

www. karger.com 